\title{
Application of gas-cooled Accelerator Driven System (ADS) transmutation devices to sustainable nuclear energy development
}

\author{
A. Abánades ${ }^{\text {a,* }}$, C. García ${ }^{b}$, L. García ${ }^{b}$, A. Escrivác ${ }^{c}$, A. Pérez-Navarro ${ }^{c}$, J. Rosales ${ }^{b}$ \\ a ETSII/Universidad Politécnica de Madrid, J.Gutiérrez Abascal, 2-28006 Madrid, Spain \\ ${ }^{\mathrm{b}}$ Instituto Superior de Tecnologia y Ciencias Aplicadas. Quinta de los, Molinos, Ave. Salvador Allende y Luaces, Ciudad de la Habana, CP 10400, Apartado Postal 6163, Cuba \\ ${ }^{\mathrm{c}}$ Instituto de Ingeniería Energética, Universidad Politécnica de Valencia, C.P. 46022 Valencia. Spain
}

\begin{abstract}
A B S T R A C T
The conceptual design of a pebble bed gas-cooled transmutation device is shown with the aim to evaluate its potential for its deployment in the context of the sustainable nuclear energy development, which considers high temperature reactors for their operation in cogeneration mode, producing electricity, heat and Hydrogen. As differential characteristics our device operates in subcritical mode, driven by a neutron source activated by an accelerator that adds clear safety advantages and fuel flexibility opening the possibility to reduce the nuclear stockpile producing energy from actual LWR irradiated fuel with an efficiency of $45-46 \%$, either in the form of Hydrogen, electricity, or both.
\end{abstract}

\section{Introduction}

Hydrogen is considered as an energy carrier capable to substitute oil and other fossil fuels enabling a sustainable energy scenario in the medium term (Penner, 2006). If such substitution is done with free- $\mathrm{CO}_{2}$ technologies, would allow for a reduction in the green house gases (GHG) emissions making possible to reach the objectives of the Kyoto Agreement and, in the case of countries without fossil fuel resources, to alleviate its dependence on foreign supplies, in many cases located in geopolitical unstable areas that make this dependence even more undesirable (Commission of the European Communities, 2006). High priority for a successful application of the hydrogen instead of oil appears in the transport sector (Ball and Wietschel, 2009), where hybrid or electric vehicles based on fuel cells fed by hydrogen, could be the way to reach above mentioned goals.

Nevertheless, the generation of the amount of hydrogen needed for fossil fuel substitution will ask for big quantities of thermal energy or electricity which might be sustainable (Midilli and Dincer, 2007) and could imply an increase in the consumption of primary energy, given the losses in the needed energy transformation, in relation to the direct use of oil for the same applications. In addition, there will be a constraint in the way to generate electricity: it should be a clean method, without GHG emissions (Pérez-Navarro et al., 2008). For keeping the goal to avoid such emissions it is of the main reasons to introduce hydrogen in the energy scenario.

In conclusion, there is a need for massive hydrogen generation methods with both low $\mathrm{CO}_{2}$ emission and low primary energy demand. Accelerator Driven Transmutation Systems (ADS), postulated to treat the long live radioactive wastes generated by the existing nuclear power plants, have the potential to fulfill with the two above mentioned conditions for hydrogen generation given there is no $\mathrm{CO}_{2}$ emissions in this nuclear process and the needed primary energy is coming from the depleted Uranium and transuranics (TRU) already existing in the radioactive wastes. In addition, its use will alleviate the environmental problem provoked by those wastes by transmuting them to less harmful wastes with much lower lifetime and reducing its mass and volume.

This paper addresses the viability of an ADS Transmutation device that uses as fuel the wastes coming from the existing LWR power plants, encapsulated in graphite, in the form of pebble beds, cooled by helium (which enables high temperatures, in the order of $1200 \mathrm{~K}$ ) to generate hydrogen either by high temperature electrolysis (Hino et al., 2004) or by thermochemical cycles (Shinji Kubo et al., 2004). Previously, other authors have suggested a separated approach in which Hydrogen is produced by critical high temperature gas reactors, producing wastes that could be diminished by a nuclear transmutation system (Tamawaki et al., 2007). We propose an integrated approach in which the transmutation step itself is used for hydrogen generation. Such a system could be operated in an autonomous way, given its capability to generate the electricity and the heat required to produce hydrogen without the need of any external supply. Besides, it could be used as back up system for the electrical network during peak demand situations. 
This paper addresses the study of a Transmutation Advanced Device for Sustainable Energy Applications (TADSEA) for simultaneous transmutation and hydrogen generation based on a graphite-gas configuration already described in (Abánades and Pérez-Navarro, 2007). Section 2 shows the hydrogen generation and transmutation scheme of the proposed pebble bed device. Section 3 introduces the computational tools used for the neutronic calculations of the device core, benchmarking them with previous existing codes, and the results obtained with those tools. Some radiological discussions regarding our proposal are developed in Section 4 . Section 5 analyses the thermal-hydraulics behavior of the device. Finally, in Section 6, these results are used for our concept of the performance of the installation for hydrogen production.

\section{Conceptual design of the proposed nuclear based Hydrogen generation scheme}

The hydrogen production from nuclear heat is one of the options under study to reach the target of generating free- $\mathrm{CO}_{2}$ hydrogen and many projects has been initiated for that purpose (Verfondern and Von Lensa, 2005), exploring various methods to use nuclear heat or electricity for hydrogen generation, including high temperature thermochemical processes (Harvego et al., 2006), or high temperature electrolysis (Utgikar and Thiesen, 2006). The hydrogen production scheme evaluated in this paper is based on a gas-cooled subcritical pebble-bed transmutation concept (Abánades and Pérez-Navarro, 2007) driven by a proton accelerator. Such nuclear device is coupled to a thermochemical I-S process (Kasahara et al., 2007a), a technological option that has been studied in detail for nuclear-based hydrogen production and that fits well to gas coolant technology.

The main components of the system are the following:

- A proton accelerator, which drives the neutron source that maintains neutronic population in the nuclear system.

- A subcritical core, composed by TRISO particle introduced in graphite pebbles, arranged in a subcritical configuration surrounding a high performance neutron spallation source and cooled by a gas.

- A hydrogen generation installation, based on the implementation of an I-S process.

- A Brayton cycle, for electricity production.

The starting conceptual nuclear design (Abánades and PérezNavarro, 2007) is based on an experimental device of a few MW conceived as candidate for a once-trough transmutation scheme called pebble-bed transmutation (PBT). For its application to Hydrogen generation, the dimensions of the gas-cooled pebble bed subcritical core have been enlarged for the new design in order to reach $100 \mathrm{MW}$ of thermal power. Table 1 shows the basic parameters of our design.

The proton accelerator technological choice is based on a linear accelerator (LINAC), instead of the cyclotron technology used for the previous few-MW experimental pebble-bed concept (Abánades and Pérez-Navarro, 2007), as the required increase in beam energy and current, following the directives of projects as the European PDS-XADS (Mueller, 2005), suggests a $1 \mathrm{GeV}-10 \mathrm{~mA}$ particle accelerator for subcritical core with a neutron multiplication factor $k<0.95$.

The subcritical core is composed by ceramic pebbles of $6-\mathrm{cm}$ diameter containing the TRISO coated particles with the fuel (in our analysis with the nuclide fraction corresponding to a LWR discharge). The gas coolant is Helium, with an outlet temperature of $950^{\circ} \mathrm{C}$, as required for a hydrogen production scheme based on the
Table 1

Basic parameters of the TADSEA.

\begin{tabular}{|c|c|}
\hline Core parameters & Value \\
\hline Internal radius $(\mathrm{cm})$ & 15.5 \\
\hline External radius $(\mathrm{cm})$ & 125.75 \\
\hline Height $(\mathrm{cm})$ & 293.94 \\
\hline Total volume $\left(\mathrm{m}^{3}\right)$ & 14.38 \\
\hline Fuel volume $\left(\mathrm{m}^{3}\right)^{\mathrm{a}}$ & 10.64 \\
\hline Reflector thickness (cm) & 60 \\
\hline Number of pebbles & 94092 \\
\hline Keff & 0.943 \\
\hline Ksrc & 0.966 \\
\hline Power $(\mathrm{MW})^{\mathrm{b}}$ & 100 \\
\hline Gain & 29.32 \\
\hline Core inlet temperature $\left({ }^{\circ} \mathrm{C}\right)$ & 590 \\
\hline Core outlet temperature $\left({ }^{\circ} \mathrm{C}\right)$ & 950 \\
\hline Coolant & Helium \\
\hline Coolant massflow $(\mathrm{kg} / \mathrm{s})$ & 53 \\
\hline Coolant pressure (MPa) & 7 \\
\hline Pumping power (MW) & 5 \\
\hline \multicolumn{2}{|l|}{ Accelerator parameters } \\
\hline Beam current $(\mathrm{mA})$ & 3.41 \\
\hline Beam energy (MeV) & 1000 \\
\hline Beam power (MW) & 3.41 \\
\hline Electricity consumption (MW) & 6.82 \\
\hline \multicolumn{2}{|l|}{ Hydrogen production } \\
\hline Cycle & $\mathrm{I}-\mathrm{S}$ \\
\hline Production $(\mathrm{kg} / \mathrm{s})$ & 0.39 \\
\hline Energy production (MW) & 46.8 \\
\hline \multicolumn{2}{|l|}{ Electricity production } \\
\hline Cycle & Brayton regenerative \\
\hline Cycle efficiency & 0.45 \\
\hline Electricity production (MW) & $45 \mathrm{MW}$ \\
\hline
\end{tabular}

a Assuming a honeycomb disposition of the balls that provides a parking factor of 0.64 .

${ }^{b}$ Power in the device with an average power density of $7 \mathrm{~W} / \mathrm{cm}^{3}$.

I-S thermochemical process in cogeneration mode with electricity production by a Brayton cycle.

\section{Neutronic analysis}

The neutronic analysis has as main objectives the calculation of the distribution of the energy production in the fuel, basic input for the thermal analysis and the cooling regime of the system, and the isotope evolution in the fuel to evaluate the radioactive waste elimination and the reduction of its final radiological hazard.

The MCNPX 2.6e code (Gregg McKinney et al., 2007) has been chosen to simulate the neutronic behavior of the TADSEA. MCNPX is a multipurpose reference Monte Carlo code that is extensively used for the simulation of Accelerator Driven System (ADS) as it merges the medium/high energy physics involved in the accelerator induced neutron source and the physics models required for neutron transport in a critical or subcritical core.

Another MCNPX.2.6e new capability used for this study is the incorporation of the predictor-corrector technique for the burn-up calculation. This method uses CINDER90 (Wilson et al., 1999) to calculate burn-up just up to the middle of the selected time step and, at this time point, calculates the neutron flux density and the reaction rates assuming steady state conditions. With the new fluxes and collision densities calculated with this approach, the burn-up is recalculated for the end of the time step considered. Using the fluxes and collision densities deduced from a steady state at the center of the time step, the average magnitude values are being considered to calculate the variation in the isotope composition along the time step. This technique allows the use of longer time step without significant precision loss in the results.

New features have been added to this Monte Carlo simulation system, incorporating CINDER90 and MonteBurns (Poston and Trellue, 1999) codes that facilitate the calculation of isotopic evolu- 
Table 2

Initial transuranic composition of the fuel (Abánades and Pérez-Navarro, 2007).

\begin{tabular}{lc}
\hline Isotope & Proportion (\%) \\
\hline $\mathrm{Np}-237$ & 4.4 \\
$\mathrm{Pu}-238$ & 1.3 \\
$\mathrm{Pu}-239$ & 51.3 \\
$\mathrm{Pu}-240$ & 23.8 \\
$\mathrm{Pu}-241$ & 7.9 \\
$\mathrm{Pu}-242$ & 4.8 \\
$\mathrm{Am}-241$ & 5.1 \\
$\mathrm{Am}-242$ & 0.9 \\
$\mathrm{Cm}-244$ & 0.16 \\
\hline
\end{tabular}

tion. Nevertheless, MCNPX burn-up estimation is limited to critical configurations and cannot be used directly for isotopic evolution analysis in a subcritical device. In our simulations the burn-up in the subcritical core is calculated by the evaluation of the average neutron flux at each zone to a single energy group from the eigenvalues problem analysis. CINDER90 works with 63 energy groups and the transversal cross sections for these 63 groups are condensed using a generic spectrum. This approach can produce some discrepancies in the final inventory of the isotopes generated from the fission products. Nevertheless, when comparing different configurations this limitation does not introduce significant errors.

This estimation method has been qualified against previous neutronic analysis that was performed with a calculation scheme based on MCNP and ORIGEN for the analysis of the PBT (Abánades and Pérez-Navarro, 2007). Both simulation approaches were used assuming a homogenous composition for the pebble fuel using the adequate mass fractions for C, Si and fuel. It was also assumed that the fuel microparticles were embedded in a graphite matrix and that all the volume left free by the fuel or the SiC was filled by graphite with a $1.7 \mathrm{~g} / \mathrm{cm}^{3}$ density. The fuel composition regarding transuranic content is shown in Table 2 as it will come from direct isotope partitioning, excluding Uranium. Cross sections for the most relevant isotopes, $\mathrm{C}$ in particular, were processed to take into account the thermal working conditions and the resonance broadening.

PBT operation procedure assumed for the simulation includes an initial step where each level is filled with fresh fuel and, in cycles of 99 days, each layer is moved to a lower level, introducing new fuel on the top layer and extracting the balls from the bottom one. With this scheme, the fuel is burned up during 990 days. After 10 cycles the system reaches composition equilibrium, where at the beginning of the new 11 th cycle there is fresh fuel in the first/upper layer. For the last/lower one, the fuel has passed a complete 99 days cycle for each of the previous cycles, having completed 891 burning days. Core was not only divided in ten horizontal layers, but also in ten concentrically cylinders with the inner one containing the spallation target, as outlined in Fig. 1.

Despite the different methodology, with different cross section libraries and codes, results show a good agreement with previous analysis, giving confidence in the use of the new version for MNCPX for the neutronic analysis in the conceptual design of TADSEA, as can be observed in Figs. 2-4.

Once the neutronic design tool has been qualified, the pebblebed core was redesigned to increase the thermal power respect to the previous concept (Abánades and Pérez-Navarro, 2007) for the application of our device to hydrogen generation. The new design is based on the enlargement of the core's geometrical dimensions (either radii or height) conserving the transmutation capabilities and power density profiles of the system, with the purpose of obtaining $100 \mathrm{MW}$ of thermal power keeping as design limit a mean power density of $7 \mathrm{~W} / \mathrm{cm}^{3}$, which is considered a typical design value for gas cooled reactors.

The main parameters of the upgraded core are shown in Table 3. We have kept the same space for the spallation target, and we have

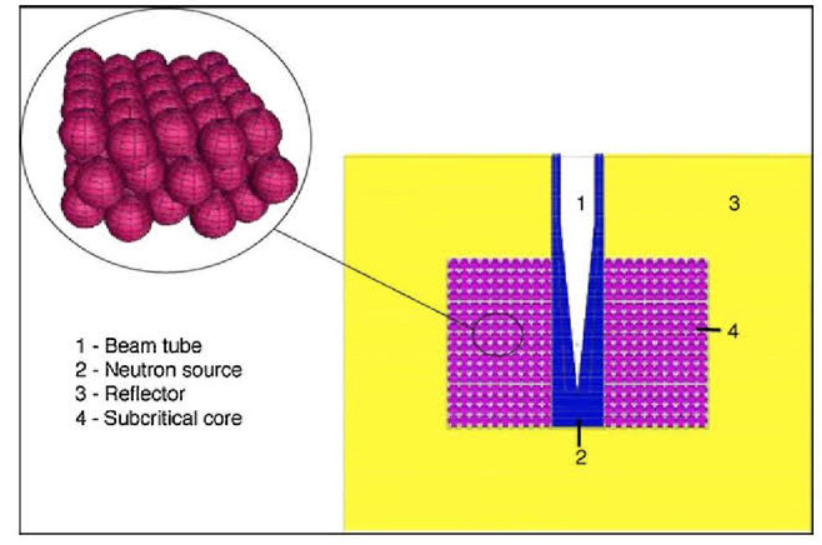

Fig. 1. Configuration of the pebble-bed subcritical device.

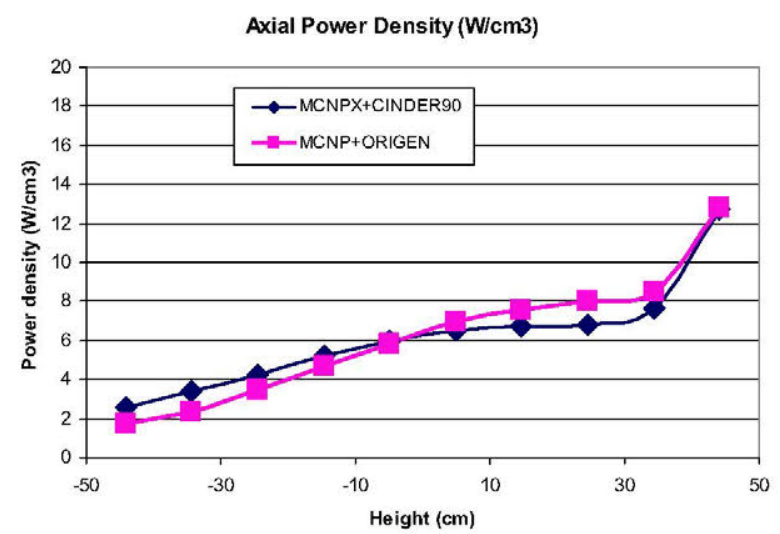

Fig. 2. Axial power density profile in the PBT (Harvego et al., 2006) using MCNPX and the MCNP+ORIGEN.

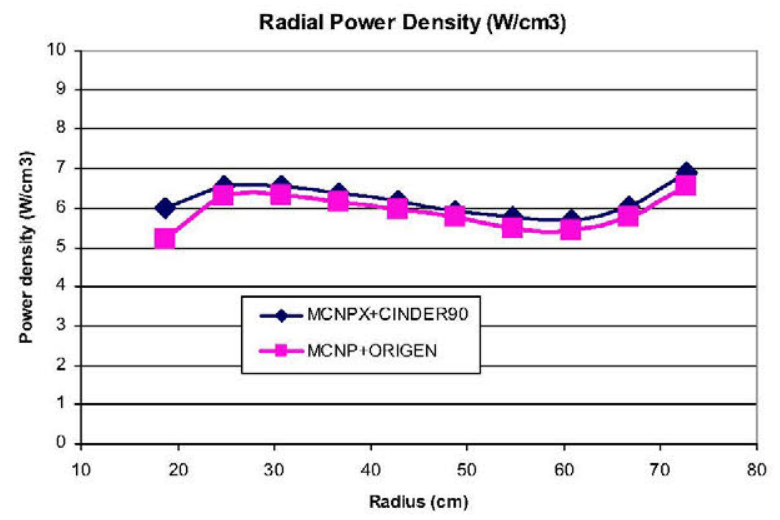

Fig. 3. Radial power density profile in the PBT (Harvego et al., 2006) using MCNPX and the MCNP+ORIGEN.

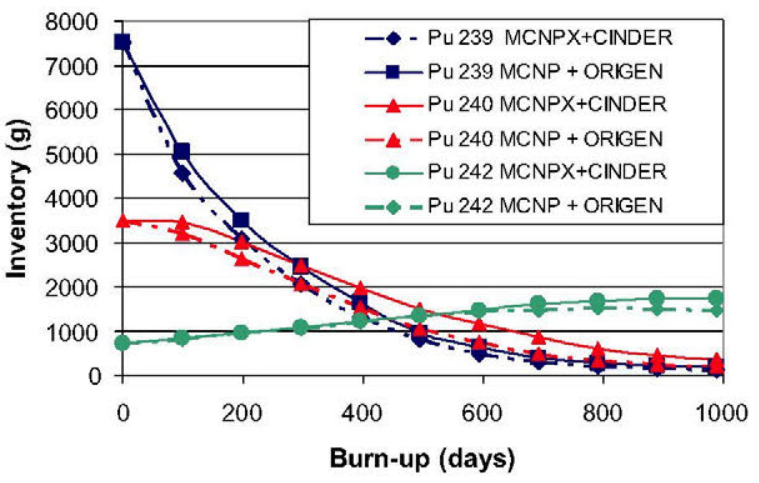

Fig. 4. Results for the evolution of Pu isotopes in PBT in our benchmarking exercise. 
Table 3

Core configuration of the $100 \mathrm{MW}$ gas-cooled pebble-bed core.

\begin{tabular}{lc}
\hline Core parameters & Value \\
\hline Internal radius $(\mathrm{cm})$ & 15.5 \\
External radius $(\mathrm{cm})$ & 125.75 \\
Height $(\mathrm{cm})$ & 293.94 \\
Total volume $\left(\mathrm{m}^{3}\right)$ & 14.38 \\
Fuel volume $\left(\mathrm{m}^{3}\right)^{\mathrm{a}}$ & 10.64 \\
Number of balls & 94092 \\
Keff & 0.9203 \\
Allowed power $(\mathrm{MW})^{\mathrm{b}}$ & 100 \\
Maximum power $(\mathrm{MW})^{\mathrm{c}}$ & 210.9
\end{tabular}

a Assuming a honeycomb disposition of the balls that provides a packing factor of 0.64 .

b Power in the device with an average power density of $7 \mathrm{~W} / \mathrm{cm}^{3}$.

c Power obtained using full accelerator capabilities: $1 \mathrm{GeV}$ and $10 \mathrm{~mA}$ on a fresh fuel.

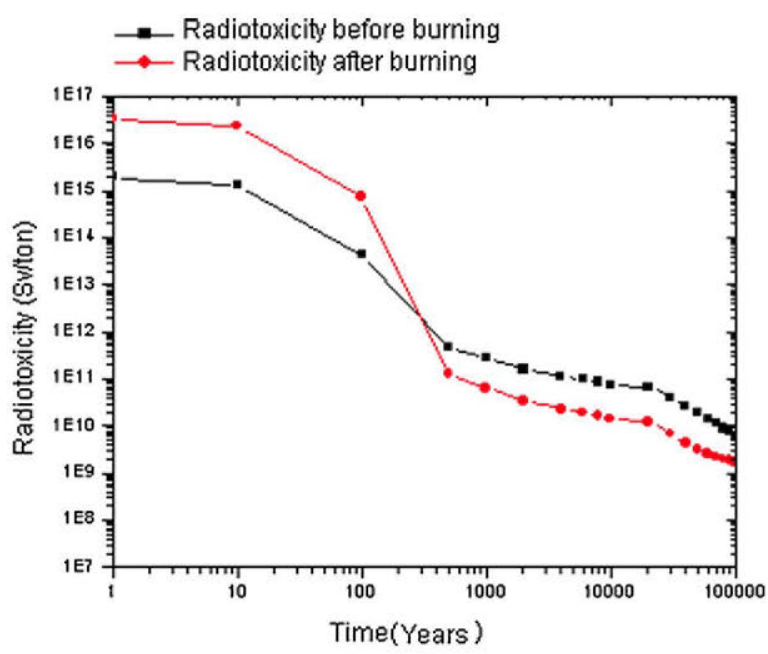

Fig. 5. Radiotoxicity reduction after TADSEA operation.

increased the core volume to $14.38 \mathrm{~m}^{3}$ to keep the mentioned mean power density. Effective neutron multiplication (Keff) is well below 0.95 even at Beginning of Operation (BO, when the core is filled with fresh fuel pebbles) to keep safety operation.

\section{Radiological analysis}

Besides the two main objectives of the system for hydrogen generation (no $\mathrm{CO}_{2}$ emissions and no increase in the demand for primary energy), there is also a third goal oriented to improve nuclear sustainability: the transmutation of long live radioactive waste from the LWR fission power plants to diminish its radiotoxicity. In order to evaluate how effective is the proposed device in transmuting, we have calculated the evolution of the radiotoxicity for the wastes in the initial load of the device and the corresponding one to the elements obtained in the discharge of the waste after a complete cycle of 990 days in the system. For radiotoxicity calculation normalized respect to the initial mass of the fuel loaded in the device, both the initial isotopes and those with significant lifetimes generated along the cycle were taken into account. Fig. 5 shows the evolution of the radiotoxicity by inhalation for both: the charge and the discharge of the system. There is a relative radiotoxicity increase at the discharge during the first 200 years, but after this time the tendency reverses and the reduction on one order of magnitude is obtained at the discharge in relation to the initial load (Figs. 6 and 7).

A detailed analysis of the responsible isotopes for this radiotoxity was completed and main findings are shown in Fig. 8 where

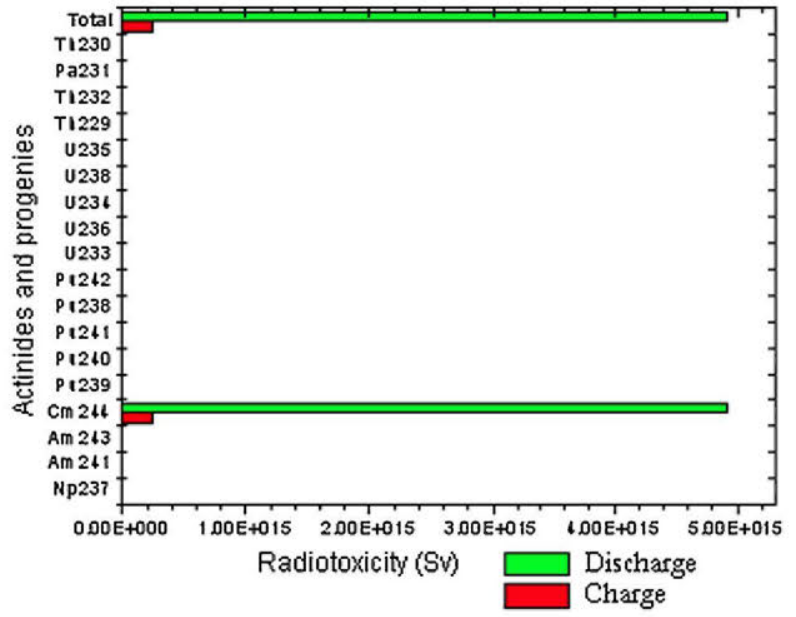

(a) $\mathrm{T}=0$

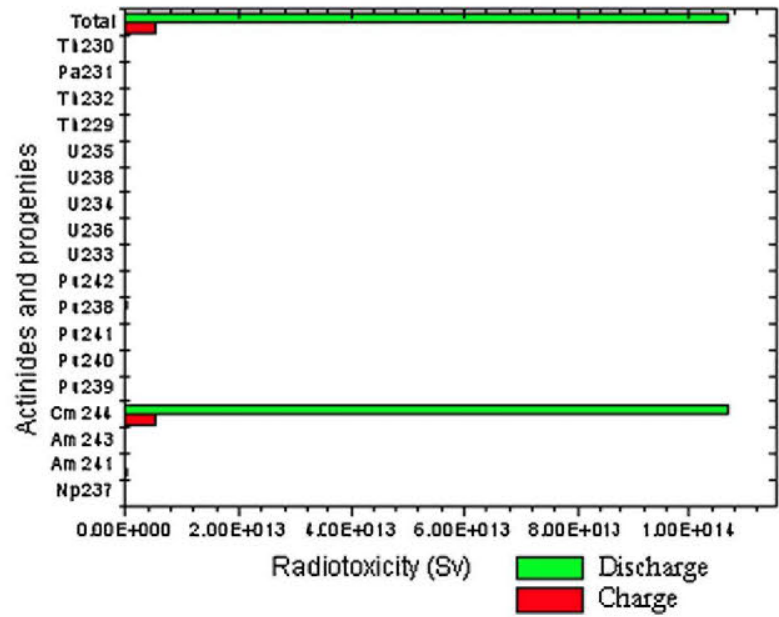

(b) $T=100 y$

Fig. 6. Radiotoxicity of heavy elements at different times from discharge of the TADSEA versus its charge.

inhalation radiotoxicity of each radioisotope at charge and discharge are compared. The initial increase is due to the generation of $\mathrm{Cm}^{244}$, but this situation changes around 200 years later, when a significant reduction in the level of $\mathrm{Pu}^{239}$ and $\mathrm{Pu}^{240}$ at the discharge allows a reduction in total radiotoxicity. Main contributors, successively at times, 500,1000, 10,000 and later, are $\mathrm{Am}^{241}, \mathrm{Pu}^{239}$ and $\mathrm{Pu}^{240}$ and $\mathrm{Th}^{229}$, respectively.

These results suggest that additionally to energy generation, it is possible to reduce the radiotoxicity of the wastes from nuclear plants. The thermal power obtained from this transmutation process can be used for different purposes. The high temperature that might be reached in ceramic fueled nuclear cores opens the possibility for its application to hydrogen generation, electricity production and/or process heat for industrial applications.

\section{Thermal-hydraulics}

The thermal analysis of the TADSEA must fulfill safety requirements based on the maximum allowed temperature in the ceramic fuel pebble that could take for granted fuel integrity, proposing a thermal-hydraulic scheme that evacuates the nuclear heat produced in the subcritical core with outlet coolant temperatures that could provide heat at the exergy required for the downstream thermochemical process. On the other hand, the maximum temperature allowed in the heat exchanger materials limits the 


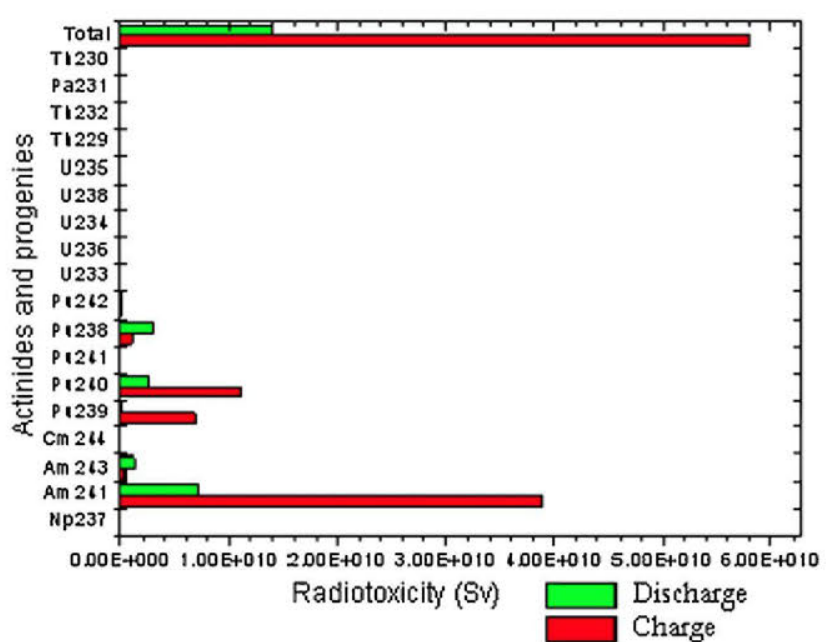

(a) $\mathrm{T}=500 \mathrm{y}$

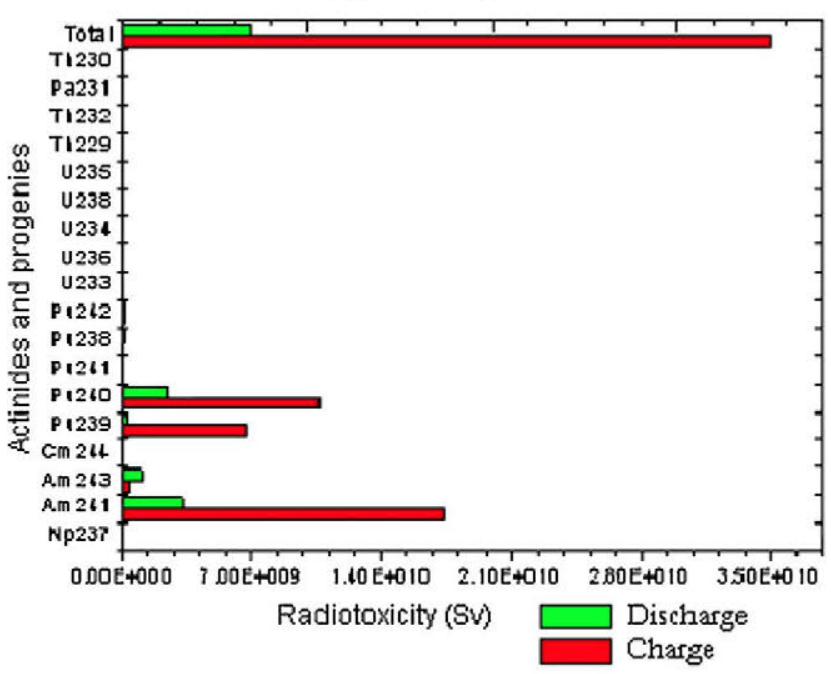

(b) $T=1000 y$

Fig. 7. Diagram of the I-S process.

temperature at the outlet of the core. Therefore, an outlet coolant temperature of $950^{\circ} \mathrm{C}$ was selected, as is proposed in the work carried out by authors from many international research projects (Harvego et al., 2006; Kunitomi et al., 2004).

A thermo-hydraulic analysis has been done in three main scenarios: at Beginning of Operation (BO), when the core is filled with fresh fuel at the first loading, at Beginning of $\mathrm{Cycle}$ (BOC), when discharge layers have completed a burn-up cycle and are substituted by fresh fuel, and at End of Cycle (EOC), after the 99 days burn-up period between refuelling. The power density distribution in the fuel provided by the neutronic analysis and shown in Figs. 2 and 3 at the BOC has been the input for our analytical evaluation of the temperature evolution in the core. A 1-D analysis based on the energy balance in the core leads to the simple relation:

$\dot{Q}=\dot{m} \times c_{\mathrm{p}} \times\left(T_{0}-T_{\mathrm{i}}\right)$

where $\dot{Q}$ is the power of the core, $c_{\mathrm{p}}$ the Helium specific heat at the mean core temperature, and $T_{0}=950^{\circ} \mathrm{C}$ and $T_{\mathrm{i}}=590^{\circ} \mathrm{C}$ as coolant outlet an inlet temperatures, as required for hydrogen production. The Helium mass-flow $\dot{m}$ with this process temperature setpoints, commonly used in the design of high temperature gas reactors, should be $53 \mathrm{~kg} / \mathrm{s}$ at an inlet pressure of $7 \mathrm{MPa}$. In this concept evaluation we have not done a complete 3-D analysis of

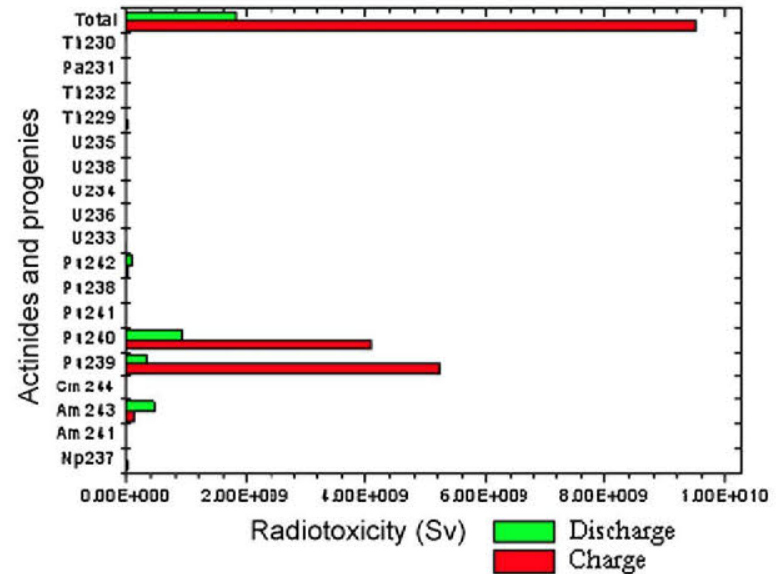

(a) $\mathrm{T}=1000 \mathrm{y}$

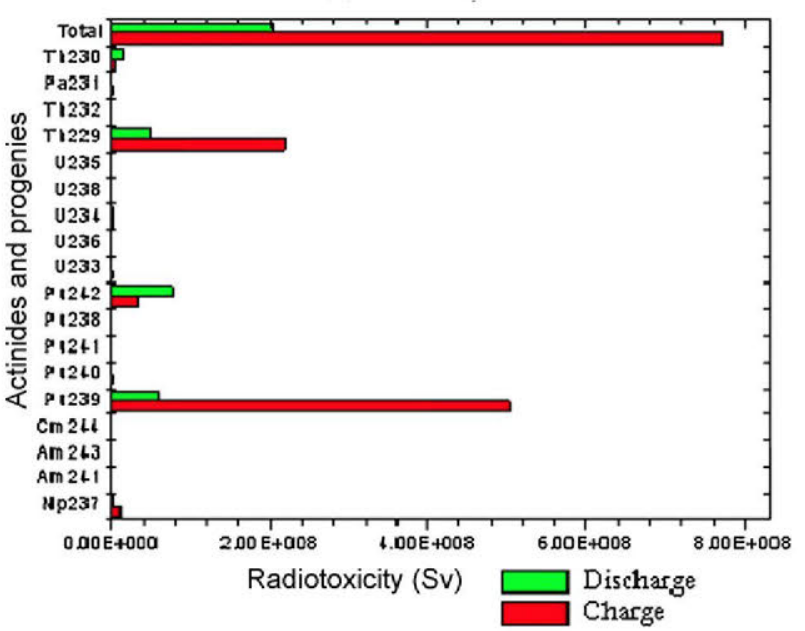

(b) $\mathrm{T}=1000 \mathrm{y}$

Fig. 8. Radiotoxicity of heavy elements at different times from discharge of the TADSEA versus its charge after 10,000 and 100,000 years.

the thermal-hydraulic of our core, that will require a 3-D computational fluid dynamic (CFD) modelling of a basic pattern of the core and a 2-D porous media analysis, to get a detailed temperature mapping of the fuel and the coolant. Nevertheless, we believe that safety operation of this pebble bed core is taken for granted as similar studies with higher outlet Helium temperature $\left(1273 \mathrm{~K},+50^{\circ} \mathrm{C}\right.$ in comparison to our design) (Nickel et al., 2002; Maki et al., 2007; Talamo et al., 2004) reported fuel temperatures below $1600 \mathrm{~K}$, maximum allowed in this fuel type, even under accidental conditions.

The Kugeler-Schulten correlation has been selected for the pressure drop estimation in the porous model of the core. Friction factors are slightly different from that calculated by the Ergun correlation for low and intermediate Reynolds number but remains more applicable to regions with high flow in which our device operates (Gao and Shi, 2002).

The Kugeler-Schulten correlation is given below:

$\Delta P=\psi \frac{(1-\varepsilon) H}{2 \varepsilon^{3} d_{\mathrm{p}} \rho_{\mathrm{f}}}\left(\frac{m_{\mathrm{f}}}{A}\right)^{2}$

$\Psi=\frac{320}{(\operatorname{Re} / 1-\varepsilon)}+\frac{6}{(\operatorname{Re} / 1-\varepsilon)^{0.1}}$,

$R e=\frac{\left(m_{\mathrm{f}} / A\right) d_{\mathrm{p}}}{\eta}, \quad 100 \leq \operatorname{Re} /(1-\varepsilon) \leq 10^{5}$ 


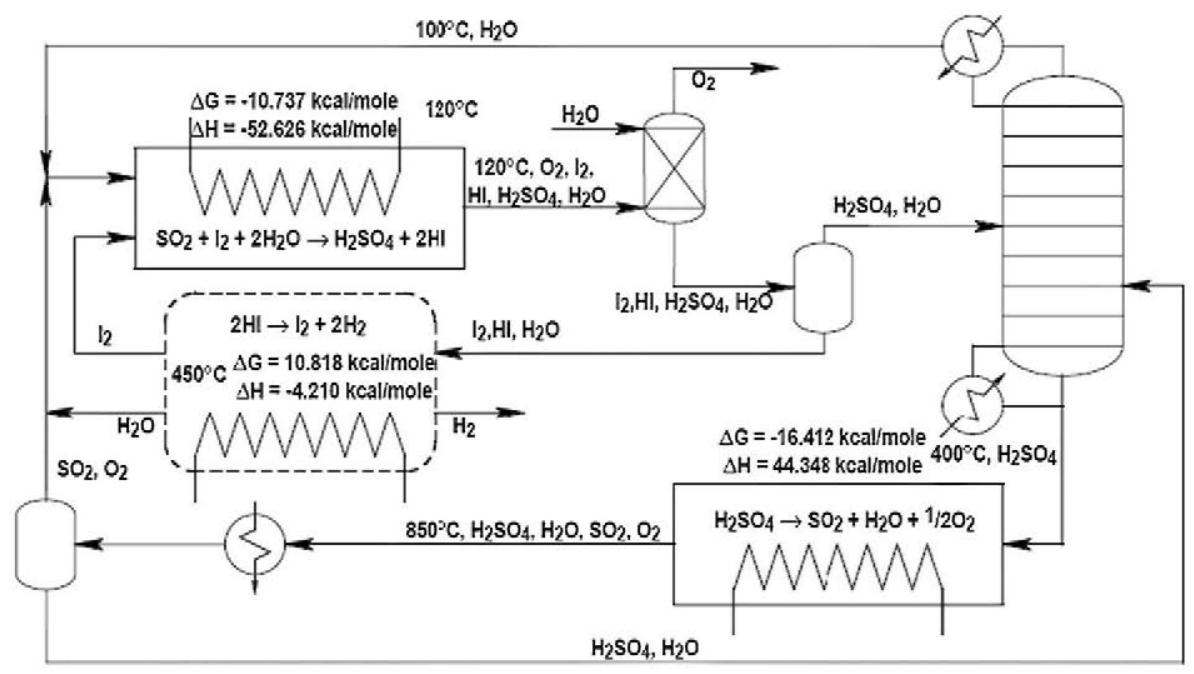

Fig. 9. Diagram of the I-S process.

where $\psi$ : friction coefficient; Re: Reynolds number; $\rho_{\mathrm{f}}$ : density of the fluid; $m_{\mathrm{f}}$ : mass flow rate of the fluid; $H$ : core height; $d_{\mathrm{p}}$ : pebble diameter; $A$ : cross-sectional area; $\varepsilon$ : porosity of the pebble bed; and $\eta$ : dynamic viscosity of the gas.

According to this correlation and with a porosity of 0.36 , for a honeycomb structure of our pebble arrangement, the pressure drop is estimated in $0.3 \mathrm{MPa}$ with a Helium mass-flow of $53 \mathrm{~kg} / \mathrm{s}$, which implies the need of $5 \mathrm{MW}$ of pumping power in the primary loop to force the coolant circulation from the top to the bottom of the core.

\section{Cogeneration scheme: Hydrogen generation and electricity production}

The application of the heat generated in the core of our subcritical device to Hydrogen production requires the selection of an adequate thermochemical process. The I-S cycle was developed in 1970 s by General Atomic and imposes thermal requirements to the design of the cooling system. A diagram of the Hydrogen production process is shown in the Fig. 9.

In this process we have the basic reactions:

$$
\begin{aligned}
& 2 \mathrm{H}_{2} \mathrm{O}(\mathrm{l})+\mathrm{I}_{2}(\mathrm{~s})+\mathrm{SO}_{2}(\mathrm{~g}) \rightarrow \mathrm{H}_{2} \mathrm{SO}_{4}(\mathrm{aq})+2 \mathrm{HI}(\mathrm{aq}) \\
& 2 \mathrm{HI}(\mathrm{g}) \rightarrow \mathrm{I}_{2}(\mathrm{~g})+\mathrm{H}_{2}(\mathrm{~g}) \\
& \mathrm{H}_{2} \mathrm{SO}_{4}(\mathrm{~g}) \rightarrow \mathrm{H}_{2} \mathrm{O}(\mathrm{g})+\mathrm{SO}_{3}(\mathrm{~g}) \rightarrow \mathrm{H}_{2} \mathrm{O}(\mathrm{g})+\mathrm{SO}_{2}(\mathrm{~g})+\frac{1}{2} \mathrm{O}_{2}(\mathrm{~g})
\end{aligned}
$$

The sulfuric acid decomposition is the most demanding in terms of thermal requirements as it is produced at $870^{\circ} \mathrm{C}$. This fact implies the need for an outlet temperature of the heat source very close to $950^{\circ} \mathrm{C}$.

At the stage of the development of our conceptual design, a general approach to the flowsheet of the hydrogen generation process is presented in this paper to evaluate the performance of this generation scheme. On the other hand, thermo-chemical processes for thermal hydrogen production are basically in a laboratory development stage. The industrial application of this process has to overcome some technological barriers as the material corrosion and durability of the equipment, as in the case of the high temperature heat exchangers in aggressive atmospheres. The chemical data for the full description and optimization of the problem is another weak point of this process, mainly for the HIx mixture (Goldstein et al., 2004; Kane and Revankar, 2008).
The thermal efficiency of the I-S process is defined as follows (Lee et al., 2009):

$\eta_{\text {th }}=\frac{\mathrm{HHV}}{Q_{\mathrm{SA}}+Q_{\mathrm{HI}}+Q_{\mathrm{P}}}$

where HHV is the high heating value for Hydrogen, $Q_{S A}$ the heat input of the sulfuric acid section, $Q_{H I}$ the heat input to the $\mathrm{HI}$ section, and $Q_{P}$ the pumping heat, that globally can be expressed as:

$\eta_{\text {th }}=\frac{\mathrm{HHV}}{\mathrm{Q}+\left(W / \eta_{\text {thb }}\right)}$

With $Q$ as the global energy input to the process from the heating source, in this case the TADSEA, and W the mechanical pumping work for the cycle flow converted to thermal power trough the corresponding thermal to work conversion efficiency $\left(\eta_{\text {thb }}\right)$.

This efficiency has been evaluated by different authors, ranging from a maximum of the order of 57-51\% (Kasahara et al., 2007b; Goldstein et al., 2005). The best estimate of the practical implementation of this process ranges from 56 to $34 \%$ (Ozturk et al., 1995; Kasahara et al., 2007b). Taken an efficiency around $47 \%$, as reported by other authors (Mathias and Brown, 2003), the amounts of Hydrogen that could be generated by our 100 MW TADSEA here described are in the order of $0.39 \mathrm{~kg} / \mathrm{s}$, that implies around 10,000 tons per year, and an equivalent power of $46.8 \mathrm{MW}$ assuming a Hydrogen Low Heating Value of $120 \mathrm{MJ} / \mathrm{kg}$.

As an alternative to the plant design it might be considered the application of a Brayton cycle, either running in parallel or in complementary mode with the Hydrogen production section, depending on the foreseen availability of the latter. A technoeconomic analysis should provide the rationale to justify the use cogeneration that could include electricity production and Hydrogen production with radioactive waste elimination. Our preliminary assessment suggests that such alternative will be acceptable in the case of a low availability of the Hydrogen production section, as it is the case of the state-of-the-art. The efficiency of the Brayton cycle in this case will reach $45 \%$ (El-Genk and Tournier, 2009), what implies a production of 45 MWe.

\section{Conclusion}

The conceptual design of a pebble bed gas-cooled transmutation device (TADSEA) has been addressed in this paper, with the purpose to show the potential this kind of new proposed systems for their deployment in the context of the sustainable nuclear energy development (www.snetp.eu). 
Such strategy considers the utilization of high temperature reactors (http://www.snetp.eu/www/snetp/images/stories/DocsAboutSNETP/sra2009.pdf) as one of the key pillars of that development as they are able to be used in cogeneration mode, producing electricity, heat and/or hydrogen. We have evaluated one of these concepts whose differential characteristics are the operation in subcritical mode, driven by a neutron source activated by an accelerator that adds clear safety advantages and fuel flexibility opening the possibility to reduce the nuclear stockpile. A special attention has been paid to the neutronic analysis of the system, applying new techniques like the use of CINDER code for subcritical systems, what has been done with a full qualification with previous confident analysis. As main conclusion, our device can produce energy from actual LWR irradiated fuel with an efficiency of $45-46 \%$, either in the form of Hydrogen, electricity, or both. Future work is intended to explore the techno-economical analysis of this system, what should include the optimization of the plant configuration.

\section{Acknowledgments}

This work has been done in the framework of technological international cooperation thanks to the MAEC-AECID grant program of the Spanish Ministry of Foreign Affairs and International Cooperation with the support of the International Cooperation Program of the UPV.

\section{References}

Abánades, A., Pérez-Navarro, A., 2007. Engineering design studies for transmutation of nuclear waste with a gas-cooled pebble-bed ADS. Nuclear Engineering and Design 237, 325.

Ball, M., Wietschel, M., 2009. The future of hydrogen-opportunities and challenges. International Journal of Hydrogen Energy 34, 615-627.

Commission of the European Communities, 2006. A European strategy for sustainable, competitive and secure energy. COM 105.

El-Genk, M.S., Tournier, J.M., 2009. Performance analices of VHTR plants with direct and indirect closed Brayton cycles and different working fluids. Progress in Nuclear Energy 51, 556-572.

The European technical working group on ADS, April 2001. A European roadmap for developing Accelerator Driven Systems for nuclear waste incineration.

Gao, Z., Shi, L., 2002. Thermal hydraulic calculation of the HTR-10 for the initial and equilibrium core. Nuclear Engineering and Design 218, 51-64.

Goldstein, S., Vitart, X., Borgard, J.M., 2004. General comments about the efficiency of the iodine-sulphur cycle coupled to a high temperature gas cooled reactor. International Scientific Journal for Alternative Energy and Ecology 3 (11).

Goldstein, S., Borgard, J.M., Vitart, X., 2005. Upper bound and best estimate of the efficiency of the iodine sulphur cycle. International Journal of Hydrogen Energy 30, 619-626.

Gregg McKinney et al., 2007. MCNPX 2.6x features (2006-2007). LA-UR-07-2053. Los Alamos National Laboratory.
Harvego, E.A., et al., 2006. An evaluation of reactor cooling and coupled hydrogen production processes using the modular helium reactor. Nuclear Engineering and Design 236, 1481-1489.

Hino, R., et al., 2004. R\&D on hydrogen production by high-temperature electrolys is of steam. Nuclear Engineering and Design 233, 363.

Kane, C., Revankar, S.T., 2008. Sulfur-lodine thermochemical cycle: HI decomposition flow sheet analysis. International Journal of Hydrogen Energy 33 5996-6005.

Kasahara, S., et al., 2007a. Flowsheet of the thermochemical water-splitting iodine sulfur process for effective hydrogen production. International Journal of Hydrogen Energy 32, 489-496.

Kasahara, S., Kubo, S., Hino, R., Onuki, K., Nomura, M., Nakao, S.I., 2007b. Flowsheet study of the thermochemical water-splitting iodine-sulfur process for effective hydrogen production. International Journal of Hydrogen Energy 32, 489-496.

Kunitomi, K., Yan, X., Shiozawa, S., Fujimoto, N., 2004. GTHTR300C for hydrogen cogeneration. In: 2nd International Topical Meeting on High Temperature Reactor Technology, Beijing, China, September 22-24.

Kyoto protocol web site: http://unfccc.int/kyoto_protocol/items/2830.php.

Lee, B.J., No, H.C., Yoon, H.J., Jin, H.G., Kim, Y.S., Lee, J.I., 2009. Development of flowsheet for iodine-sulfur thermo-chemical cycle based on optimized Bunsen reaction. International Journal of Hydrogen Energy 34, 2133-2143.

Maki, John T., et al., 2007. The challenges associated with high burnup, high tem perature and accelerated irradiation for TRISO-coated particle fuel. Journal of Nuclear Materials 371, 270-280.

Mathias, Paul M., Brown, Lloyd C., 2003. Thermodynamics of the sulphur-iodine cycle for the thermochemical hydrogen production. In: Aspen Technology Inc. General Atomics. 68th Annual meeting of the Society of Chemical Engineers, Japan , University of Tokyo, Marcj 23-25.

Midilli, A., Dincer, I., 2007. Ket strategies of hydrogen energy systems for sustainability. International Journal of Hydrogen Energy 32, 511-524.

Mueller, A.C., 2005. Nuclear waste incineration and accelerator aspects from the European PDS-XADS study. Nuclear Physics A 751, 453c-468c.

Nickel, $\mathrm{H}$, et al, 2002. Long time experience with the development of HTR fue elements in Germany. Nuclear Engineering and Design 217, 141.

Ozturk, I.T., Hammache, A., Bilgen, E., 1995. An improved process for H2SO4 decom position step of the sulfur-iodine cycle. Energy Conversion and Management 36 (1), 11-21.

Penner, S.S., 2006. Steps toward the hydrogen economy. Energy 31, 33-43.

Pérez-Navarro, A., et al., 2008. Wind energy contribution to a sustainable transport: the case of Spain. World Electric Vehicle Journal 2. (3).

Poston, D.L., Trellue, H.R., 1999. User's Manual, Version 2.0 for MONTEBURNS Version 1.0, L.A-UR-99-4999 (September).

Shinji Kubo, et al., 2004. A demonstration study on a closed-cycle hydrogen production by the thermochemical water-splitting iodine-sulfur process. Nuclear Engineering and Design 233, 347.

www.snetp.eu.

http://www.snetp.eu/www/snetp/images/stories/Docs-AboutSNETP/sra2009.pdf.

Talamo, A., et al., 2004. Key physical parameters and temperature reactivity coefiicients of the deep burn modular helium reactor fuelled with LWRs waste. Annals of Nuclear Energy 31, 1913-1037.

Tamawaki, M., et al., 2007. Application of nuclear energy for environmentally friendly hydrogen generation. International Journal of Hydrogen Energy 32 2719-2725.

Utgikar, V., Thiesen, T., 2006. Life cycle assessment of high temperature electrolysis for hydrogen production via nuclear energy. International Journal of Hydrogen Energy 31, 939-944.

Verfondern, K., Von Lensa, W., 2005. Past and present research in Europe on the production of nuclear Hydrogen with HTGR. Progress in Nuclear Energy 47, $472-483$.

Wilson, W.B., England, T.R., Van Riper, K.A., 1999. Status of CINDER '90 Codes and Data, Los Alamos National Laboratory, preprint LA-UR-99-361. 